\section{Development of multiplex DNA electronic microarrays using a universal adaptor system for detection of single nucleotide polymorphisms}

\author{
Shirley Tsang, Zhonghe Sun, Claudia Stewart, Nicole Lum, \\ Casey Frankenberger, Marianne Subleski, Lynn Rasmussen, and \\ David J. Munroe \\ SAIC-Frederick, National Cancer Institute, Frederick, MD, USA
}

BioTechniques 36:682-688 (April 2004)

The NanoChip ${ }^{\circledR}$ electronic microarray is designed for the rapid detection of genetic variation in research and clinical diagnosis. We have developed a multiplex electronic microarray assay, specific for single nucleotide polymorphism (SNP) genotyping and mutation detection, using universal adaptor sequences tailed to the $5^{\prime}$ end of PCR primers specific to each target. $P C R$ products, amplified by primers directed to the universal adaptor sequence, are immobilized on the microarray either directly or via capture oligonucleotides complementary to the universal adaptor sequence. This simple modification results in a significant increase in fidelity with improved specificity and accuracy. In addition, the multiplexing of genetic variant detection allows increased throughput and significantly reduced cost per assay. This general schema can also be applied to other microarray and macroarray formats.

\section{INTRODUCTION}

The NanoChip ${ }^{\circledR}$ Molecular Biology Workstation (Nanogen, San Diego, CA, USA) was designed for the detection of single nucleotide polymorphisms (SNPs) (1). It has also been developed for other related DNA hybridization analyses, including point mutations (2-4), short tandem repeats (5), and gene expression (6). In addition, this platform can be used for infectious disease detection, genetic and cancer diagnoses, and a variety of pharmacogenomic applications.

The complete NanoChip workstation consists of a NanoChip cartridge, a sample loader, and a fluorescence-based reader, with computer-controlled interface and data analysis software. The NanoChip cartridge, which uses a semiconductor microchip, incorporates an electronic microarray with $10 \times 10(100)$ test sites. Each individual test site is directly wired with a microelectrode located beneath the test site. Following user-defined programs, an electronic field is produced on the surface that allows charged molecules to be transported to or from any test site (7). The active electronic microarray surface is covered with an agarose or polyacrylamide hydrogel permeation layer. The permeation layer contains streptavidin, which allows binding of biotinylated oli- gonucleotides $(7,8)$. Applying an electric field to the microarray enables the immobilization and subsequent hybridization and detection of DNA at given sites on the semiconductor chip $(7,8)$. Detection is mediated via Cy ${ }^{\mathrm{TM} 3}$ - and Cy5-labeled probes for each locus/allele that hybridizes to the target DNA. The NanoChip cartridge is then washed and imaged. The ratio of $\mathrm{Cy} 3$ to $\mathrm{Cy} 5$ fluorescence signal allows for discrimination between homozygous (wild-type or mutant) and heterozygous samples.

DNA can be applied to the NanoChip microarray by either of two methods, amplicon-down or capture-down (9-12). Using the amplicon-down method, a biotinylated PCR product that is produced using biotin-labeled primers is directly addressed to the test site by interaction with streptavidin on the surface layer of the electronic microarray. Using the capture-down method, a biotin-labeled capture oligonucleotide that is specific for the target PCR product is selectively addressed and bound to the array. The capture oligonucleotide functions to facilitate the binding of target PCR products to specific pads on the array. The array is then hybridized with a set of fluorescently labeled reporter probes that are specific for each allele in the target DNA sequence. Stringent single-base mismatch discrimination is achieved thermally or electronically. In this study, we introduce a universal adaptor system that utilizes a specific universal sequence labeled with biotin to either capture or directly tag the target PCR product for immobilization onto the array. We used inbred mouse genomic DNA and two representative SNP markers from our in-house mouse SNP panel (S. Tsang and D.J. Munroe, unpublished data). The universal adaptor system was designed for universally capturing target DNA to the NanoChip microarray via either the amplicon-down or capture-down strategy. This type of universal adaptor system has been widely used in other applications, such as DNA sequence and PCR-based detecting systems (8). These simple modifications result in significantly enhanced flexibility, multiplicity, specificity, and accuracy, as well as increased throughput and reduced cost per assay. This general schema can also be applied to other microarray and macroarray formats.

\section{MATERIALS AND METHODS}

\section{Target Amplification}

The mouse genomic DNA of Balb/ CByJ, Balb/CJ, DBA/1J, DBA/2J, 129/J, NOD/Lt, 129S1/SvImJ, SPRET/Ei, SCAST/Ei, C57BL/6J, A/JCr, 129J/ $\mathrm{NCr}, \mathrm{CBA} / \mathrm{NCr}, \mathrm{AKR} / \mathrm{NCr}, \mathrm{C} 3 \mathrm{H} / \mathrm{MeJCr}$, FVB/NCr， C57BL/6NCr， SJL/NCr were purchased from The Jackson Laboratory (Bar Harbor, ME, USA). Two SNP markers from our in-house mouse SNP panel (S. Tsang and D.J. Munroe, unpublished data) were selected and used to develop our multiplex DNA electronic array using a universal adaptor system. Two PCR primer sets, TS013 and TS028, were used in the assay (Table 1). The flanking sequences to the $5^{\prime}$ end of the underlined sequences of TS013F and TS028F are M13 forward (fM13) primer sequences (see Figure 2), and the flanking sequences of TS013R and TS028R are M13 reverse (rM13) primer sequences (see Figure 2). PCR product lengths were 334 bp for the TS013 marker and 391 bp for the TS028 marker. Biotinylated fM13 primer and biotinylated rM13 primer (Table 1) were used in the amplicon-down format. Biotinylated fM13 
Table 1. Oligonucleotides Used in Universal Adaptor System for SNP Detection

\begin{tabular}{|ll|}
\hline Primer & Sequence \\
\hline fM13 primer & Biotin-5'-GTAAAACGACGGCCAGT-3' \\
rM13 primer & Biotin-5'-GGAAACAGCTATGACCATG-3' \\
fM13 capture & 5'-ACTGGCCGTCGTTTTAC-3'-biotin \\
oligonucleotide & \\
rM13 capture & 5'-CATGGTCATAGCTGTTTCC-3'-biotin \\
oligonucleotide & \\
TS013F & 5'-GTAAAACGACGGCCAGTAATGGGAAAAGCAGATGGATG-3' \\
TS013R & 5'-GGAAACAGCTATGACCATGATGGTACAGGGGTCTCTGGTG-3' $^{\prime}$ \\
TS013 stacker & 5'-GCCAATGTCCAGCATGCTAGCAGAT-3' \\
TS013 reporter & Cy3-5'-GGCTATCATTTTTAT-3'/Cy5-5'-GGCTATCATTTTTAA-3' \\
TS028F & 5'-GTAAAACGACGGCCAGTAGTATCGAGGGCTGCTACAGG-3' \\
TS028R & 5'-GGAAACAGCTATGACCATGGCCTTTGTGTCTTCCTGACAA-3' \\
TS028 stacker/ & 5'-ACCGATCCGCGAATCCGGAA-3'/ \\
stablizer & 5'-GTCCGGCCTCGATTAAGGAG-3' $^{\prime}$ \\
TS028 reporter & Cy3-5'-GCCGGAAATGA-3'/Cy5-5'-GCCGGAAATGG-3' \\
The underlined region of the primer is specific to the target DNA. SNP, single nucleotide polymorphism. \\
\hline
\end{tabular}

capture oligonucleotide and biotinylated rM13 capture oligonucleotide were used in the capture-down format (Table 1). All primers and oligonucleotides were synthesized by Integrated DNA Technologies (Coralville, IA, USA).

PCR amplification was performed using $10 \mathrm{ng}$ of genomic mouse DNA, 1× High-Fidelity PCR buffer (Invitrogen,
Carlsbad, CA, USA), $1.5 \mathrm{mM} \mathrm{MgSO}_{4}$, $100 \mathrm{nM}$ each of the pair of primers, 200 $\mu \mathrm{M}$ each of the four dNTPs, and $1 \mathrm{U}$ Platinum ${ }^{\circledR}$ Taq High Fidelity DNA polymerase (Invitrogen) in a total volume of $25 \mu \mathrm{L}$. The reactions were heated at $95^{\circ} \mathrm{C}$ for $3 \mathrm{~min}$, followed by 40 cycles at $95^{\circ} \mathrm{C}$ for $1 \mathrm{~min}, 55^{\circ} \mathrm{C}$ for $1 \mathrm{~min}$, and $72^{\circ} \mathrm{C}$ for $1 \mathrm{~min}$, and then incubated at $72^{\circ} \mathrm{C}$ for 10

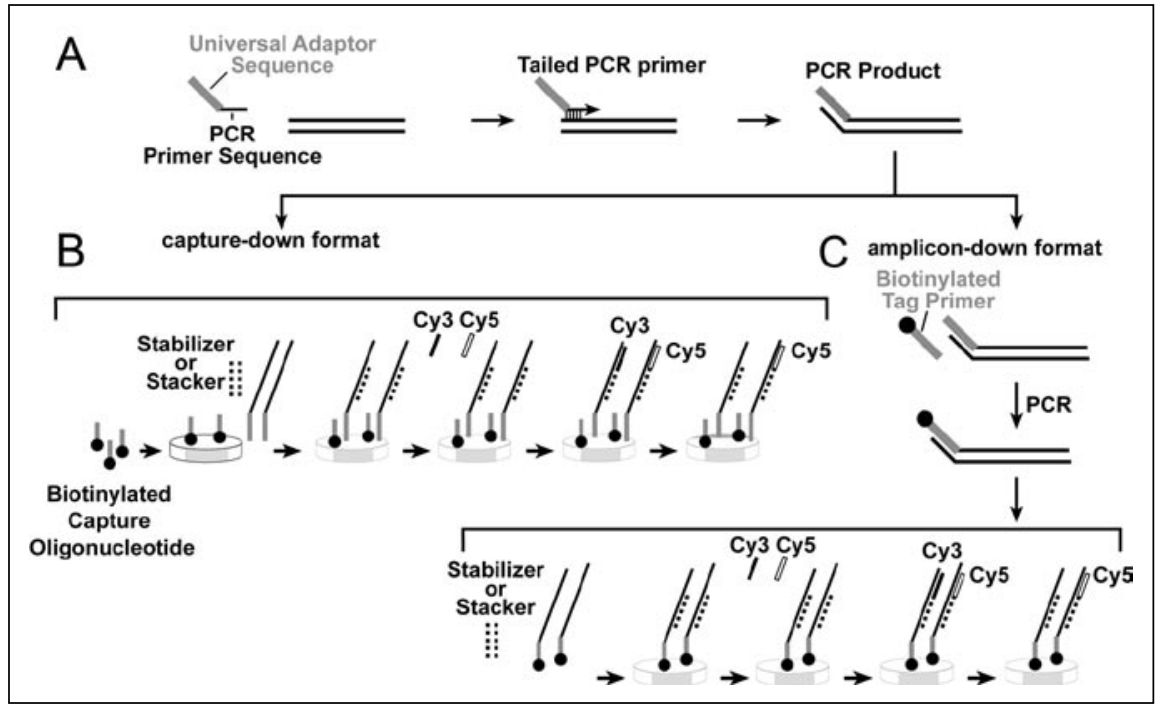

Figure 1. Assay scheme of capture-down and amplicon-down formats using universal adaptor sequence. (A) PCR primer tailed with universal adaptor sequence is used to amplify the target DNA. (B) In the capture-down format, the biotinylated capture oligonucleotides complementary to the universal adaptor sequence are electronically deposited to the test site to capture the tailed-target DNA with the universal adaptor sequence. The stabilizer and/or stacker oligonucleotides are used in the discrimination of the two allelic reporters labeled with $\mathrm{Cy} 3$ or Cy5. (C) In the amplicon-down format, the biotinylated tag primer is used to tag the amplified target DNA in PCR amplification. The tagged PCR product is directly addressed to the test site along with the stabilizer and/or stacker oligonucleotides, followed by detection of the two reporters labeled with Cy3 and Cy5, respectively. 
min in a PTC-225 Peltier Thermal Cycler (MJ Research, Waltham, MA, USA).

\section{Target PCR Product Preparation}

The PCR products were desalted using the Sephadex ${ }^{\circledR}$ G-50 system (Millipore, Billerica, MA, USA) prior to addressing to the NanoChip cartridge. The 96-well Sephadex G-50 plate was prepared by first pouring the $5-\mathrm{mL}$ Sephadex G-50 powder (Fluka, St. Louis, MO, USA) onto a MultiScreen ${ }^{\circledR}$ column loader (Millipore). The residual powder was then scraped off the loader surface. A MultiScreen filter plate (Millipore) was aligned on top of the column loader and then flipped quickly. The Sephadex G-50 powder was hydrated with $300 \mu \mathrm{L}$ of distilled water for $1 \mathrm{~h}$. Excess water was cleared from the plate, and the G50 columns were packed by centrifugation at $1000 \times g$ for $2 \mathrm{~min}$. The columns were washed twice by adding $150 \mu \mathrm{L}$ of water, followed by centrifugation at $1000 \times g$ for $2 \mathrm{~min}$. Ten microliters of PCR product were added to each well, and the plate was centrifuged at $1000 \times$ $g$ for $2 \mathrm{~min}$. The desalted samples were collected in a new 96-well plate. The purified DNA was quantitated by gel electrophoresis using the National Institutes of Health (Bethesda, MD, USA) Image $1.62(13,14)$ The amplified samples were diluted with water (final concentration of 5-25 $\mathrm{nM}$ ) and mixed with an equal volume of $100 \mathrm{mM}$ histidine (total volume of $60 \mu \mathrm{L}$ for 1 cartridge) in a 96-well plate. Stacker and stabilizer oligonucleotides were added to the PCR product (TS013

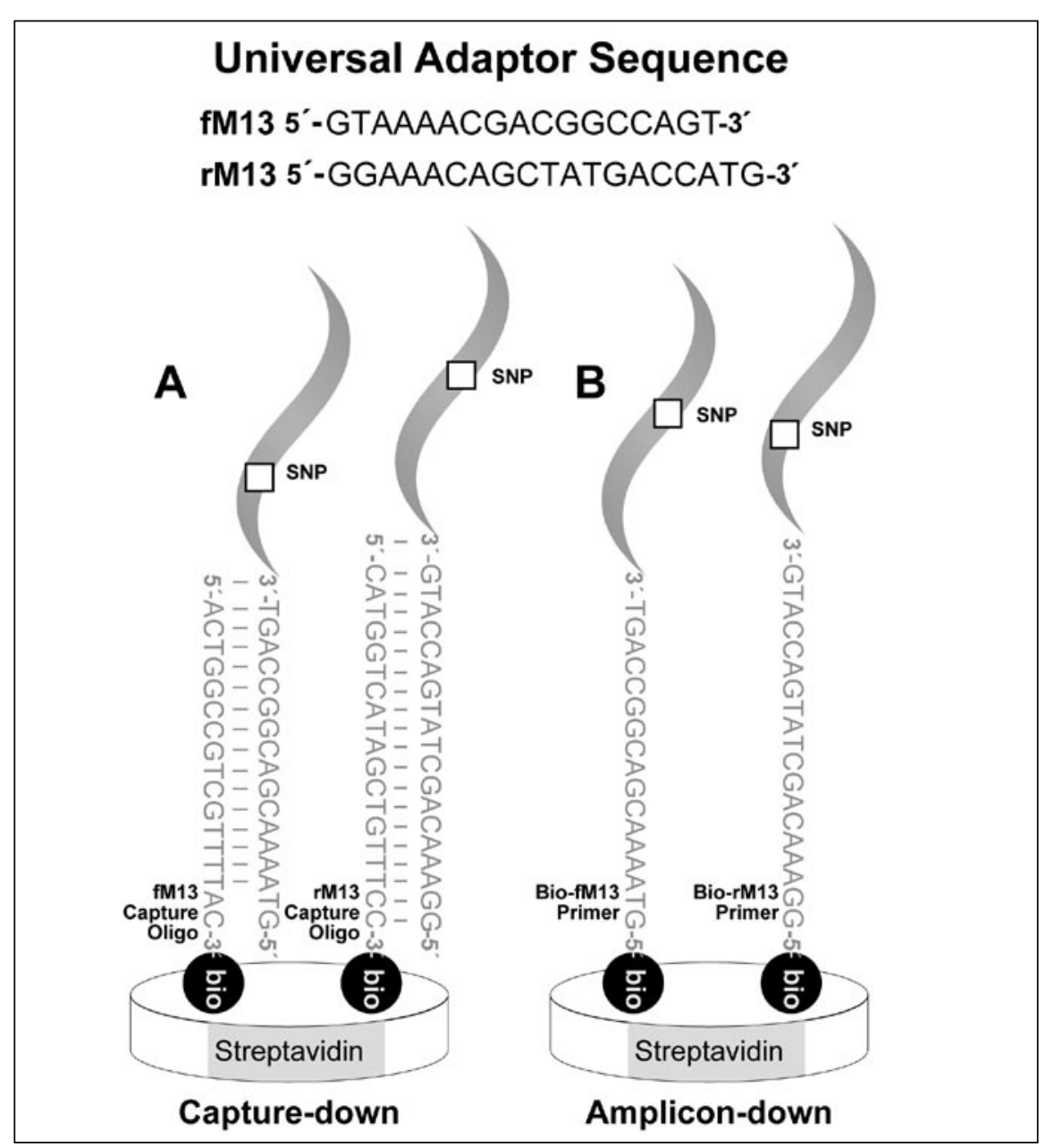

Figure 2. Sequences of universal adaptor fM13 and rM13. (A) The binding of the universal adaptor sequence fM13 and rM13 tailed at target DNA with biotinylated fM13 capture oligonucleotide and rM13 capture oligonucleotide, respectively, in the capture-down method. (B) Biotinylated universal adaptor sequence fM13 and rM13 are tagged at amplified target DNA in the amplicon-down method. SNP, single nucleotide polymorphism; bio-, biotinylated; rM13, M13 reverse primer sequence; fM13, M13 forward primer sequence. stacker, TS028 stacker, and TS028 stabilizer; see Table 1). The stacker oligonucleotide was used to assist in reporter discrimination using base stacking energy-transfer techniques (Nanogen. 2000. NanoChip standard SNP research application note no. 140083.1). The stabilizer oligonucleotide was used to remove any target secondary structures around the SNP site. $(5,16,17)$. The concentration of the biotinylated captures (biotinylated fM13 capture oligonucleotide and biotinylated rM13 capture oligonucleotide), the stackers, and the stabilizer was 500 $\mathrm{nM}$; the target concentration was 5-25 $\mathrm{nM}$. The conductivities of the samples were approximately $60 \mu \mathrm{S} / \mathrm{cm}$. The target solutions were heated to $95^{\circ} \mathrm{C}$ for 5 min to denature the target and remove the secondary structures with the presented stabilizer and/or stacker. The targets were kept on ice prior to loading.

\section{Target Sample Loading}

The NanoChip cartridge was pretreated with $0.3 \mathrm{M} \mathrm{NaOH}$ for $5 \mathrm{~min}$, and then rinsed with water or $50 \mathrm{mM}$ histidine. The treatment of $0.3 \mathrm{M}$ $\mathrm{NaOH}$ can also be used to dissociate the binding of two nucleotide oligonucleotides. The 96-well plate (containing the desalted targets) and the NanoChip cartridge were placed in the NanoChip Molecular Biology Workstation's loader for loading. A loader file was created to electronically address specific samples to user-defined test sites. The capture address was set to $60 \mathrm{~s}$ for the addressing of the M13 adaptors (biotinylated fM13 capture oligonucleotide and biotinylated rM13 capture oligonucleotide). The target address was set to $120 \mathrm{~s}$ for the addressing of the biotinylated PCR products. The activation voltage used to capture the M13 adaptor was determined by the number of pads to be activated to reach an activation level of 400-500 nA/pad. The target activation was set at $2 \mathrm{~V}$ for one pad (approximately $450 \mathrm{nA} / \mathrm{pad}$ ). In addition to the loading the targets, a nontarget control (histidine buffer) and a heterozygous control were loaded for each assay. After loading, the cartridge was washed with $500 \mu \mathrm{L}$ of water and rinsed with $150 \mu \mathrm{L}$ of high-salt buffer (50 mM sodium phosphate, $\mathrm{pH} 7.4$, $500 \mathrm{mM} \mathrm{NaCl}$ ). 


\section{Probe Hybridization}

A pair of Cy3- and Cy5-labeled allele-specific oligonucleotide reporters (Table 1) were mixed at $1 \mu \mathrm{M}$ each in $100 \mu \mathrm{L}$ of high-salt buffer and hybridized to the cartridge for $5 \mathrm{~min}$ at room temperature. The cartridge was washed with $150 \mu \mathrm{L}$ of high-salt buffer to stop the hybridization.

\section{Sample Detection and Analysis}

Sample detection and analysis were performed as an automated function on the NanoChip Molecular Biology Workstation's reader. Allelic discrimination was determined using ratios of the two fluorescently labeled allele-specific reporter oligonucleotides that had been normalized to a heterozygous control. Data were generated using thermal strin- gency. A thermal reader file ramped from $28^{\circ} \mathrm{C}$ to the predetermined discrimination temperature in two-degree steps. At each step, the cartridge was kept at a specific temperature for $60 \mathrm{~s}$, washed, and scanned using the reader's two-laser system for fluorescence intensity. Depending on the fluorescence intensity, the accumulation duration and signal gain level were adjusted (NanoChip standard SNP research application note).

All inbred mouse strains have been backcrossed for at least 20 consecutive generations and should be isogonic. The F1 of C57BL/6J and SPRET/Ei are TA heterozygote at TS013 locus and AG heterozygote at TS028 locus with $50 \%$ of each genotype. These two heterozygotes were used in every assay to normalize the two $\mathrm{Cy} 3$ and Cy5 fluorescently labeled reporters. Normalization of signal was critical in determining the

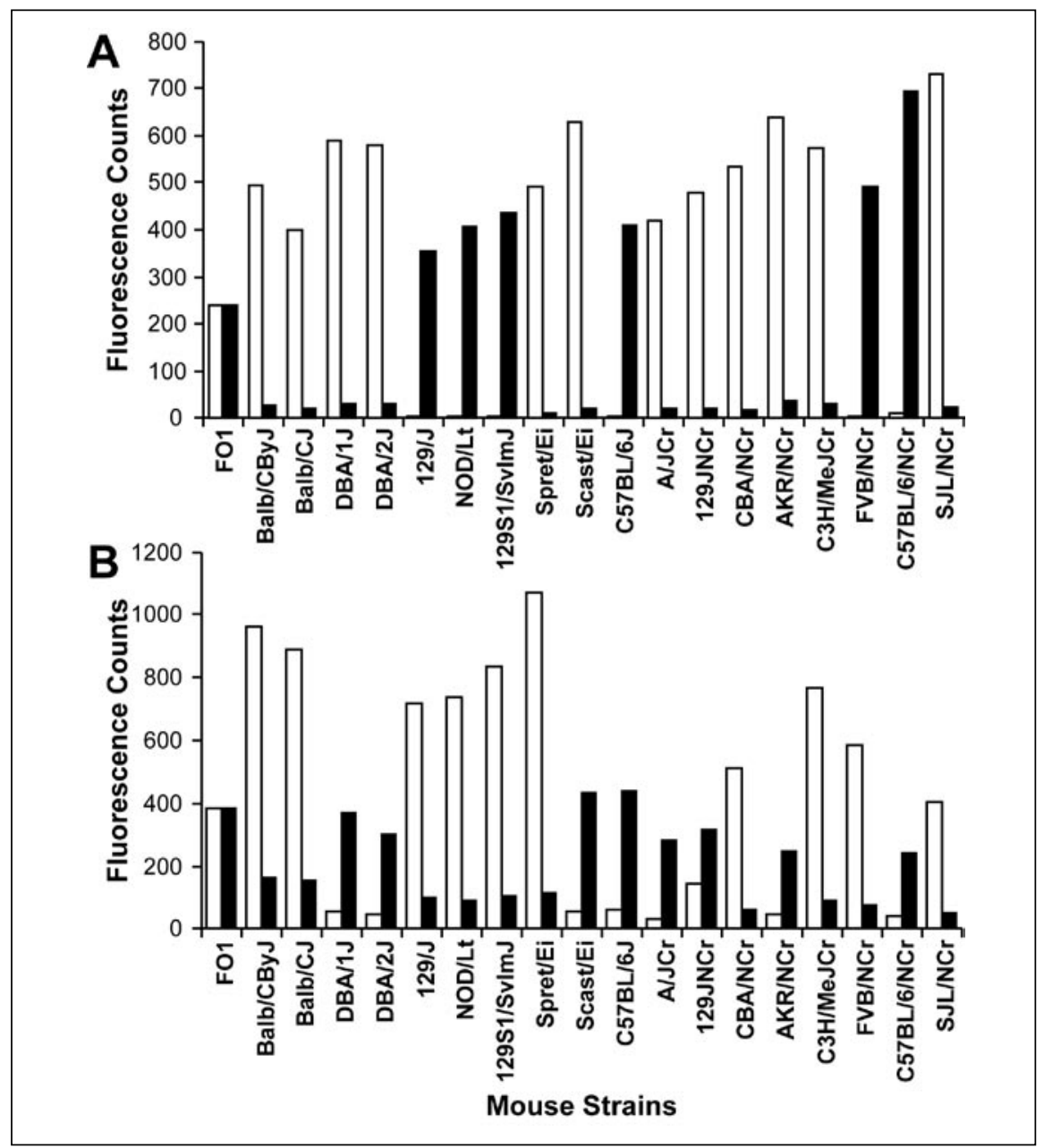

Figure 3. Genotype results for 18 inbred mouse strains at selected loci. (A) TS013 (TA) locus and (B) TS028 (AG) locus. The F01 is the hybrid of C57BL/6J and SPRET/Ei. The dark bar represents C57BL/6J, and the open bar represents SPRET/Ei. 
genotype of an SNP. At the end stage $\left(50^{\circ}-52^{\circ} \mathrm{C}\right)$, the fluorescence counts were close to the background control. The cartridge was rinsed with $150 \mu \mathrm{L}$ of high-salt buffer and then probed with the second pair of reporters, which hybridized to the other set of targets already contained on the microarray.

\section{RESULTS AND \\ DISCUSSION}

\section{Experiment Design}

In our system, target DNA is first amplified by specific PCR primers tailed with a universal adaptor sequence at the $5^{\prime}$ end (Figure 1A). An oligonucleotide, complementary in sequence to the universal adaptor, is labeled with biotin to capture different target DNA that are amplified by primers tailed with the universal adaptor sequences (Figure 1B). Alternatively, an oligonucleotide with the universal adaptor sequence can be directly labeled with biotin at the $5^{\prime}$ end and used in a second round of PCR to tag the target PCR product tailed with the universal adaptor sequence (Figure 1C). We have used M13 forward or reverse sequences as our universal adaptor (Figure 2). These M13 forward and reverse adaptor sequences are used to tail the $5^{\prime}$ end of forward and reverse targetspecific PCR primers, respectively. In the capture-down strategy, biotinylated capture oligonucleotides, complementary to the fM13 adaptor sequence or rM13 adaptor sequence, are used as universal capture oligonucleotides to immobilize targets onto NanoChip test sites via a biotinylated tag that interacts with streptavidin in the microarray permeation layer. In the amplicon-down method, one of the M13 forward and reverse primers is biotinylated at the $5^{\prime}$ end and used in a nested PCR to amplify PCR products tailed with the fM13 or the rM13 adaptor sequences. The biotinylated prod- uct from the second PCR, mixed with stacker and stabilizer oligonucleotides, is then electronically addressed to a test site on the NanoChip cartridge. The stacker and stabilizer oligonucleotides are used to enhance hybridization and increase signal $(5,16,17)$. Following the immobilization of target sequences onto the test sites, a pair of $\mathrm{Cy} 3-$ and Cy5-labeled reporters is applied to the microarray (Figure 1, B and C), and the cartridge is then scanned for fluorescence detection.

To test the universal adaptor strategy for use in SNP genotyping, two SNP markers, TS013 and TS028 (S. Tsang and D.J. Munroe, unpublished data), were typed on 18 inbred mouse strains. Genomic DNA of these inbred mouse strains was amplified with PCR primers tailed with M13 adaptor sequences (TS013F/TS013R and TS028F/TS028R) and genotyped by both the capture-down and amplicon-down strategies.

\section{Capture-Down}

Biotinylated fM13 and rM13 capture oligonucleotides (Figure 2A) were both addressed to a single test site. Mouse genomic DNA amplified with TS013 or TS028 specific primers tailed with M13 universal adaptor sequence(s) was then captured at the test sites via the capture oligonucleotides. The TS013 product tailed with the fM13 sequence and the TS028 product tailed with rM13 tail sequence were simultaneously captured on the same test site as the detecting strands. Control DNA (C57BL/6J, SPRET/Ei, C57BL/6J $\times$ SPRET/Ei) and a no target control were always loaded on the same NanoChip cartridge. The resulting TS013 and TS028 genotypes for the 18 mouse strains are displayed in Figure 3, A and B, respectively.

Theoretically, when using the capture-down method, target DNA can be released from the biotinylated capture oligonucleotide by dissociating the binding between the M13 capture oligonucleotide and the M13 tailed sequences of target (including the reporter, stacker, and stabilizer) using 0.3 $\mathrm{M} \mathrm{NaOH}$, which allows for the reuse of the NanoChip cartridge. To demonstrate reuse, the array used in the experiment shown in Figure 3 was treated with 0.3 $\mathrm{M} \mathrm{NaOH}$ to "melt" off all targets and probes. A second set of identical tar-

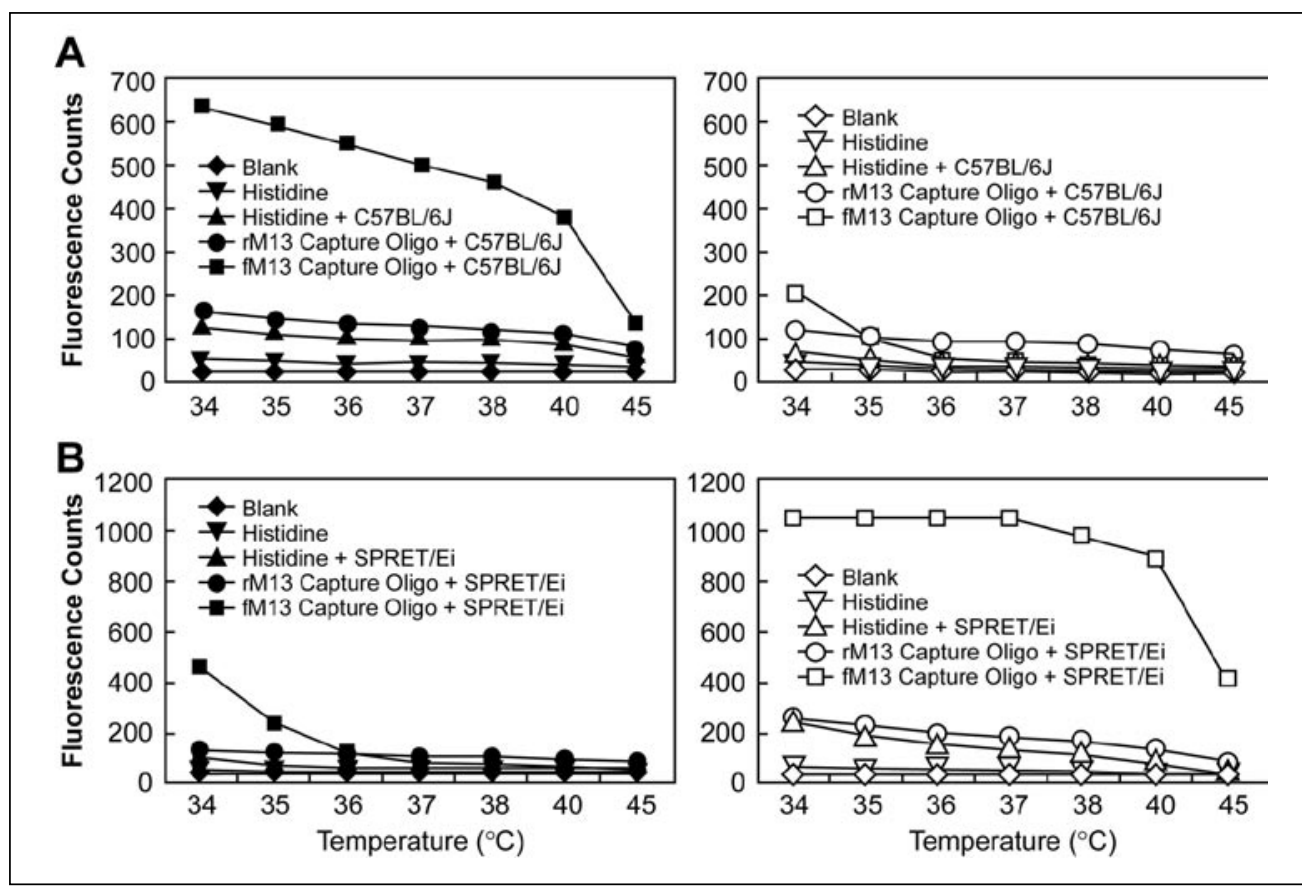

Figure 4. The specificity of capture oligonucleotide used in the capture-down method. (A) Fluorescent count of the melting curve for the inbred C57BL/6J mouse. (B) Fluorescent counts for the inbred SPRET/Ei mouse. The Cy3 and Cy5 fluorescence were normalized by fluorescent counts of hybrid of C57BL/6J $\times$ SPRET/Ei. The detecting strand of TS013 is tailed with fM13 adaptor sequence. The filled lines are the Cy3-labeled allelic report specifically for C57BL/6J, and the open lines are the Cy5-labeled reporter for SPRET/Ei. rM13, M13 reverse primer sequence; fM13, M13 forward primer sequence; oligo, oligonucleotide; +, loading. 
gets and probes were readdressed to the same array in a different order. The genotypes generated from the second load were identical to those of the first load (data not shown). These results indicate that the NanoChip array could be reused, thus increasing the flexibility and multiplicity of the microarray. We have found that the NanoChip cartridge can be reused at least three times without accumulated background noise or reduced signal (S. Tsang and Z. Sun, data not shown).

To test the specificity of the universal adaptor system, fM13 and rM13 capture oligonucleotides were used to capture C57BL/6J and SPRET/Ei TS013 PCR products tailed with the M13 universal adaptor sequence(s). Biotinylated fM13 and rM13 capture oligonucleotides were addressed to three test sites separately. Each of the three test sites was individually addressed with TS013 PCR products tailed with the fM13 adaptor sequence derived from C57BL/6J, SPRET/Ei, and C57BL/6J $\times$ SPRET/Ei hybrid DNA. The fM13-tailed DNA was also addressed separately to another three control sites in the absence of the fM13 and rM13 capture oligonucleotides. Cy3 and Cy5 allele-specific TS013 reporters were then applied to the array (Figure 4). There was no signal detected in the absence of the fM13 and rM13 capture oligonucleotides. The rM13 capture oligonucleotide was unable to capture the detecting strand of TS013 because the detecting strand of loci TS013 was tailed with fM13 sequence. All samples were correctly typed for TS013 with no cross-reactivity from the rM13-tailed strand of the PCR products.

\section{Amplicon-Down}

Mouse DNA, corresponding to 18 inbred strains, was amplified in a two-step PCR assay. The first round of amplification was with TS013- or TS028-specific primers (tailed with M13 adaptor sequences), followed by a second round with forward and reverse M13 primers that had been biotinylated at the $5^{\prime}$ end (Figure 1C). TS013 primary PCR products were amplified in the second round with biotinylated M13 forward primers and nonbiotinylated M13 reverse primers. The TS028 products were amplified in the second round with nonbiotinyl- ated M13 forward primers and biotinylated M13 reverse primers. The resulting second-round amplification products were addressed to the test site by direct binding to streptavidin in the permeation layer of the microarray. The single-stranded products from the TS013and TS028-tailed amplifications, with biotinylated fM13 and rM13 adaptor sequences, respectively, were bound to a test site in a mixture including stabilizer and stacker. The resulting genotypes identified by this procedure are identical to those shown in Figure 3. All genotypes shown in the study were confirmed by direct sequencing of genomic DNA with $100 \%$ concordance (S. Tsang and D.J. Munroe, unpublished data).

\section{Advantage of Using the Universal Adaptor System on the NanoChip Electronic Microarray}

The universal adaptor system has been used with a variety of technology platforms, such as sequencing multiple PCR fragments with a universal sequence primer tailed at the PCR primers to simplify the workflow (8). Here we highlight a number of advantages of using the universal adaptor system over the current amplicon-down and capture-down methods when applied to the Nanogen platform, such as $(i)$ reduced cost, (ii) increased flexibility, (iii) improved reagent quality control, and (iv) potential for multiplexing.

First, this universal adaptor approach dramatically reduces costs associated with the purchase of oligonucleotides. Instead of labeling each individual PCR primer with biotin for the amplicondown method or labeling each targetspecific probe with biotin for the capture-down method, the target region can be amplified by a pair of "cold" or nonbiotin-labeled PCR primers tailed with the universal adaptor sequences. The biotinylated fM13 and rM13 capture oligonucleotides can capture the PCR products of the target region by direct association with the fM13 and rM13 universal adaptor sequences. The PCR products of the target region can also be amplified by a biotin-labeled fM13 or rM13 primer in a second-round PCR and then addressed on the test site.

Second, the universal adaptor approach enhances the flexibility of current 
methods for addressing target samples. Amplified products with a universal tailed sequence can be addressed to the NanoChip cartridge by either the amplicon-down or the capture-down method.

Third, because the quality of the biotin label is critical, the universal adaptor approach reduces inconsistencies associated with variability introduced in the manufacturing and synthesis of the biotinylated primers. A single batch of biotin-labeled oligonucleotide can be tested, used, and stored at $-20^{\circ} \mathrm{C}$ for a significant period of time.

Finally, the universal adaptor system allows the NanoChip cartridge to be reused. A single cartridge can be stripped and reloaded at least three times without reduced signal strength or increased background. The universal adaptor system in conjunction with the Nanogen platform provides users with a viable, alternative method of SNP detection.

\section{ACKNOWLEDGMENTS}

This work was supported by National Cancer Institute grant no. NO1-CO12400. Our thanks to Mrs. Maritta Grau for the correction of the manuscript and to Mrs. Ellen Frazier for graphic assistance [Science Applications International Corporation (SAIC)-Frederick, Frederick, MD, USA].

\section{REFERENCES}

1.Gilles, P.N., D.J. Wu, C.B. Foster, P.J. Dillon, and S.J. Chanock. 1999. Single nucleotide polymorphic discrimination by an electronic dot blot assay on semiconductor microchips. Nat. Biotechnol. 17:365-370.

2.Pollak, E.S., L. Feng, H. Ahadian, and P. Fortina. 2001. Microarray-based genetic analyses for studying susceptibility to arterial and venous thrombotic disorders. Ital. Heart J. 2:568-572.

3.Kudaravalli, R., T. Tidd, M. Pinotti, A. Ratti, R. Santacroce, M. Margaglione, B. Dallapiccola, F. Bernardi, et al. 2002. Polymorphic changes in the $5^{\prime}$ flanking region of factor VII have a combined effect on promoter strength. Thromb. Haemost. 88:763-767.

4.Evans, J.G. and C. Lee-Tataseo. 2002. Determination of the factor $\mathrm{V}$ Leiden singlenucleotide polymorphism in a commercial clinical laboratory by use of NanoChip microelectronic array technology. Clin. Chem. 48:1406-1411.

5.Radtkey, R., L. Feng, M. Muralhidar, M. Duhon, D. Canter, D. DiPierro, S. Fallon, E. Tu, et al. 2000. Rapid, high fidelity analysis of simple sequence repeats on an electronically active DNA microchip. Nucleic Acids Res. 28:E17.

6.Weidenhammer, E.M., B.F. Kahl, L. Wang, M. Duhon, J.A. Jackson, M. Slater, and X. Xu. 2002. Multiplexed, targeted gene expression profiling and genetic analysis on electronic microarrays. Clin. Chem. 48:18731882.

7.Heller, M.J., A.H. Forster, and E. Tu. 2000. Active microeletronic chip devices which utilize controlled electrophoretic fields for multiplex DNA hybridization and other genomic applications. Electrophoresis 21:157-164.

8.Gurtner, C., E. Tu, N. Jamshidi, R.W. Haigis, T.J. Onofrey, C.F. Edman, R. Sosnowski, B. Wallace, et al. 2002. Microelectronic array devices and techniques for electric field enhanced DNA hybridization in low-conductance buffers. Electrophoresis 23:1543-1550.

9.Westin, L., C. Miller, D. Vollmer, D. Canter, R. Radtkey, M. Nerenberg, and J.P. O'Connell. 2001. Antimicrobial resistance and bacterial identification utilizing a microelectronic chip array. J. Clin. Microbiol. 39:1097-1104.

10.Westin, L., X. Xu, C. Miller, L. Wang, C.F. Edman, and M. Nerenberg. 2000. Anchored multiplex amplification on a microelectronic chip array. Nat. Biotechnol. 18:199-204.

11.Ewalt, K.L., R.W. Haigis, R. Rooney, D. Ackley, and M. Krihak. 2001. Detection of biological toxins on an active electronic microchip. Anal. Biochem. 289:162-172.

12.Edman, C.F., P. Mehta, R. Press, C.A. Spargo, G.T. Walker, and M. Nerenberg. 2000. Pathogen analysis and genetic predisposition testing using microelectronic arrays and isothermal amplification. J. Investig. Med. 48:93-101.

13.Goldfarb, M. 1999. Two-dimensional electrophoresis and computer imaging: quantitation of human milk casein. Electrophoresis 20:870-874.

14.Vodovotz, Y., A. Hsing, J.A. Cook, R.W. Miller, D.A. Wink, D.M. Ritt, J.B. Mitchell, and D. Danielpour. 1997. Qualitative and quantitative analysis of DNA fragmentation using digital imaging. Anal. Biochem. 250:147-152.

15.Parinov, S., B. Barsky, G. Yershov, E. Kirillov, E. Timofeev, A. Belgovskiy, and A. Mirzabekov. 1996. DNA sequencing by hybridization to microchip octa-and decanucleotides extended by stacked pentanucleotides. Nucleic Acids Res. 24:2998-3004.

16.Yershov, G., V. Barsky, A. Belgovskiy, E. Kirillov, E. Kreindlin, I. Ivanov, S. Parinov, D. Guschin, et al. 1996. DNA analysis and diagnostics on oligonucleotide microchips. Proc. Natl. Acad. Sci. USA 93:4913-4918

Received 5 December 2003; accepted 6 February 2004.

Address correspondence to Shirley Tsang, Laboratory of Molecular Technology, 915 Toll House Avenue, Suite 211, Frederick, MD 21702, USA. e-mail: shirleytsang@ncifcrf.gov 